ISBN: 978-980-427-021-5

\title{
Tipología del pie para mejorar el bienestar físico en niños futbolistas
}

\author{
Kelly Díaz Theran' ${ }^{1}$ Carlos Hoyos Espitia, Juan Aduen Ángel
}

\section{Resumen}

La práctica deportiva del futbol implica cambios a nivel morfológico y físico, que deben ser monitoreados desde las edades iniciales, a fin de asegurar las mejores condiciones para su práctica y su bienestar físico. La caracterización del tipo de pie en deportistas suele ser un problema complejo sobre el cual poco se ha ahondado, sin embargo, esta información es útil a la hora de planificar el entrenamiento y en el mejoramiento del bienestar y condiciones físicas del mismo. El presente estudio se propuso determinar la tipología del pie en niños de 12 a 14 años. El estudio se enmarcó dentro de un diseño de investigación cuantitativa con enfoque descriptivo de corte transversal utilizando un muestreo probabilístico simple para la valoración de 232 niños de la ciudad de Sincelejo a los cuales se les tomó la impresión plantar y posteriormente se analizaron los resultados en el software libre Kinovea tomando como referencia el método de Hernández Corvo para para establecer el tipo de pie. Los hallazgos encontrados muestran que la mayor proporción de los niños presentaron un pie cavo bilateral con diferencias mínimas en el lado izquierdo y el derecho. Estos resultados evidencian que el uso de la tecnología es una estrategia valiosa y eficaz para la valoración diagnóstico y posteriores intervenciones de alteraciones del pie que pueden mejorar las condiciones en las cuales se desarrolla la práctica deportiva y prevenir lesiones por sobre uso, garantizando el bienestar del niño.

Palabras clave: Tipología del pie, bienestar físico, futbol.

1 Corporación Universitaria del Caribe Cecar/Ciencias del Deporte y la Actividad Física. Sincelejo - Colombia. Correo electrónico: kelly.diaz@cecar.edu.co 


\section{INTRODUCCIÓN}

Según Gómez Salazar, L. (2010: 158), el pie del ser humano es una estructura compleja, de alta especialización de su biomecánica y funciones, las cuales le permiten cumplir las funciones de locomoción, amortiguación y equilibrio, evidenciando una adecuada distribución de cargas sobre el sistema musculo-esquelético tanto en condiciones estáticas como dinámicas: movimiento.

Así mismo Cailliet, R. (2006: 256) sustenta que en muchas ocasiones los niños que realizan práctica deportiva, especialmente fútbol pueden sentir dolor en los pies en algún momento de la práctica deportiva, debido a diferentes agentes que pueden estar asociados a factores extrínsecos del deporte como el calzado, el tipo de terreno, el mal uso de vendajes o también por factores intrínsecos como aumento en el índice de masa corporal (IMC) o tipología del pie y esto puede ver afectado la calidad de vida del deportistas en formación.

La presente investigación pretende determinar cuál es la tipología del pie de los niños que practican fútbol en las escuelas de formación deportiva en el municipio de Sincelejo Sucre, debido a que un análisis de este tipo puede prevenir distintas lesiones a temprana edad y contribuir de una manera mucho más eficiente en la preparación deportiva de estos niños, además se podría llevar un control en la formación de cada uno de estos en pro de la mejora de su bienestar físico.

Uno de los métodos usados para la evaluación y diagnóstico del pie consiste en el análisis de la huella plantar a través del método Herzco según Gómez Salazar, L. (2010: 158). Además Barajas, R. Santana, L. (2010: 148) y Mattus, J. Pérez, D. (1999: 77) se referencian el método del Doctor Roberto Hernández Corvo de 1999 la cual es ampliamente utilizada en el ámbito deportivo ya que a través de ella permite ver la relación porcentual (\%) de la anchura metatarsiana y la anchura de la bóveda plantar y obtener una clasificación del pie en normal, plano, cavo y algunas combinaciones entre estos tipos y de esta forma establecer el tipo de pie que tiene un individuo clasificándolo de acuerdo a los resultados de la fórmula matemática planteada por el autor.

Además, el análisis se realizó a través de un software de libre acceso muy utilizado en el entorno deportivo llamado Kinovea, el cual es un programa de edición de vídeo un tanto particular. No se ha diseñado para cortar y componer sino para analizar las imágenes. Para este caso en él se editaron las tomas fotográficas de las huellas plantares tomadas sobre el 
papel fax, las cuales se graficaron y arrojaron unos datos que posteriormente utilizando la fórmula de Hernández Corvo arrojo unos resultados para dar respuesta a esta investigación.

\section{MATERIALES Y MÉTODOS}

Esta investigación se realizó en la ciudad de Sincelejo capital del departamento de Sucre, Colombia, en el año 2015, con niños de 12 a 14 años de las escuelas y clubes de formación en fútbol con una población total de 693 y un muestreo probabilístico simple mediante el procedimiento de selección por números aleatorios de 232 niños (Sexo Masculino). Se construyó un marco muestral ordenado por escuela deportiva y luego los participantes se ordenaron por orden alfabético. Se definieron los siguientes estimadores:

Tabla 1. Estimadores de la muestra

\begin{tabular}{|l|c|}
\hline \multicolumn{1}{|c|}{ Estimadores } & Sincelejo \\
\hline $\begin{array}{l}\text { Población de niños de 12 a 14 años de las } \\
\text { escuelas y clubes de formación en fútbol. }\end{array}$ & 693 \\
\hline Nivel de confianza 95\%(Z) & 1,96 \\
\hline Proporción esperada de dolor podal (p) & 0,30 \\
\hline Proporción no esperada (q) & 0,70 \\
\hline Precisión (d) & 0,05 \\
\hline Tamaño de la muestra (n) & 220 \\
\hline Muestra ajustada a la pérdida ( $\mathrm{R}=5 \%)$. & 232 \\
\hline
\end{tabular}

El diseño de investigación es cuantitativo con un enfoque descriptivo es un estudio de corte transversal. Dentro de los criterios de inclusión los participantes en el estudio debían tener las siguientes características: ser hombres, con un rango de edad entre 12 a 14 años cumplidos al momento de la evaluación, estar vinculado mínimo hace un año a la escuela de formación deportiva y que realizaran mínimo una vez a la semana la práctica del futbol.

Se inició la recolección de información donde por medio del consentimiento informado se notificó y pidió permiso a los padres de familia o acudientes, los cuales aceptaron y firmaron la participación de su hijo en el estudio, además se utilizó un Instrumento (ad hoc) Registro de datos sociodemográficos e índice de masa corporal.

PROCEDIMIENTO:

- Aleatorización y reclutamiento de los niños. 
- Aceptación y firma del consentimiento informado por parte de entrenadores.

- Evaluación de variables sociodemográficas, antropométricas.

- Toma de la huella plantar.

- Aplicación del método Herzco.

- Sistematización, tabulación y traficación.

- Análisis de información, discusión de resultados y realización del informe final.

- El tiempo promedio de aplicación de los instrumentos por cada niño fue:

- Evaluación de variables sociodemográficas y antropométricas: 10 minutos.

- Toma de la huella plantar: 5 minutos por cada evaluador.

- Análisis de huella plantar en software Kinovea 10 minutos.

El presente estudio se consideró como "investigación con riesgo mínimo" de acuerdo al artículo 11 de la resolución 008430 de 1993 del Ministerio de Salud Colombiano, ya que se emplearon pruebas de evaluación clínica y de adherencia de carácter no invasivo, debidamente estandarizadas y validadas previamente por expertos, que no atentaron contra la integridad física y moral de los participantes del estudio ${ }^{1}$. La participación en el estudio fue totalmente voluntaria, previa autorización a través de la aceptación y firma de un consentimiento informado por parte de los entrenadores de los participantes. Los resultados se utilizaron solo para fines investigativos preservando los principios de integridad e intimidad de las personas. Toda la información obtenida y los resultados de la investigación fueron tratados confidencialmente y archivados en papel y medio electrónico.

Adicionalmente, esta investigación cumplió con los principios enunciados en la Declaración de Helsinki de la Asociación Médica Mundial Consejo de Organizaciones Internacionales de las Ciencias Médicas (CIOMS). (1993: 53), su interés fue científico, en todo momento se protegió la integridad de los participantes, se tomaron todas las precauciones del caso para respetar su vida privada y para reducir al mínimo el impacto del estudio en su integridad física y mental.

Por otra parte, se respetan los derechos de autor de los diferentes insumos teóricos y evaluaciones utilizadas, citando las respectivas referencias bibliográficas.

1 República de Colombia - Ministerio de Salud. Decreto 11, Resolución No 008430. Santafé de Bogotá (1993). 
En los análisis estadísticos se utilizó el software SPSS versión 18, con el cual se realizó un análisis descriptivo de variables cuantitativas determinando los mínimos, máximos, media y desviación estándar, descriptivos de variables cualitativos.

\section{RESULTADOS}

\section{ANÁLISIS UNIVARIADOS}

\section{Variables sociodemográficas}

Se evaluaron 232 niños (sexo masculino) de 12 a 14 años de 16 escuelas de formación en fútbol de la ciudad de Sincelejo, con un promedio de 7 años de escolaridad, con Índice de Masa Corporal $\left(\mathrm{k} / \mathrm{m}^{2}\right)$ con una media de 19,08 normal. La medida podométrica (Método Herzco) del pie derecho con una media de 56,01 normal, y en pie izquierdo con una media de 57,79. (Tabla 2.)

Tabla 2. Descriptivos de variables cuantitativas

\begin{tabular}{|l|c|c|c|c|c|}
\hline \multicolumn{1}{|c|}{ Variable } & N & Mínimo & Máximo & Media & $\begin{array}{c}\text { Desviación } \\
\text { estándar }\end{array}$ \\
\hline Edad (meses) & 232 & 128 & 179 & 159,96 & 10.112 \\
\hline Peso (kg) & 232 & 23 & 76 & 45.03 & 9.030 \\
\hline Talla (cm) & 232 & 130 & 177 & 153.20 & 11.104 \\
\hline $\begin{array}{l}\text { Índice de Masa Corporal (k/ } \\
\text { m2) }\end{array}$ & 232 & 9.53 & 27.57 & 19.0817 & 2.62570 \\
\hline $\begin{array}{l}\text { Medida podométrica (Mé- } \\
\text { todo Herzco) - Pie derecho } \\
\text { (0-100) }\end{array}$ & 232 & 11.58 & 84.52 & 56.0111 & 11.67546 \\
\hline $\begin{array}{l}\text { Medida podométrica (Mé- } \\
\text { todo Herzco) - Pie izquier- } \\
\text { do (0-100) }\end{array}$ & 232 & 14.29 & 94.62 & 57.7912 & 11.62250 \\
\hline
\end{tabular}

\section{Variables podométricas e IMC}

Los niños contaban con un Índice de Masa Corporal (IMC) promedio de $19,08 \mathrm{k} / \mathrm{m}^{2}$, el $80,6 \%$ clasificado como normal, el 9,5\% con sobrepeso y el $8,6 \%$ con bajo peso (tabla 3). La tipología de pie derecho predominante, medida con el Método Herzco fue el pie cavo con el 34,5\% de los casos, seguido del pie normal 29,3\% y con menor prevalencia el pie plano normal $1,3 \%$. Por su parte, la tipología de pie izquierdo predominante fue el pie cavo con el $37,5 \%$, seguido del pie normal cavo y normal con el $25,0 \%$ y con menor prevalencia el pie cavo extremo con el 1,3\% (tabla 3 ). 
Tabla 3. Descriptivos de variables cualitativas.

\begin{tabular}{|c|c|c|}
\hline Categoría & Variable & $\begin{array}{l}\text { Muestra } \\
(n=232)\end{array}$ \\
\hline \multirow[t]{21}{*}{$\begin{array}{l}\text { Podométricas } \\
\text { e IMC }\end{array}$} & Índice de Masa Corporal & \\
\hline & Bajo peso & $8.6 \%$ \\
\hline & Normal & $80.6 \%$ \\
\hline & Sobrepeso & $9.5 \%$ \\
\hline & Obeso & $1.3 \%$ \\
\hline & Tipología del pie derecho (Método Herzco) & \\
\hline & Pie plano & $6.5 \%$ \\
\hline & Pie plano normal & $1.3 \%$ \\
\hline & Pie normal & $29.3 \%$ \\
\hline & Normal cavo & $25.9 \%$ \\
\hline & Cavo & $34.5 \%$ \\
\hline & Cavo fuerte & $2.6 \%$ \\
\hline & Cavo extremo & $0 \%$ \\
\hline & $\begin{array}{l}\text { Tipología del pie izquierdo (Método Herz- } \\
\text { co) }\end{array}$ & \\
\hline & Pie plano & $5.6 \%$ \\
\hline & Pie plano normal & $2.2 \%$ \\
\hline & Pie normal & $25.0 \%$ \\
\hline & Normal cavo & $25.0 \%$ \\
\hline & Cavo & $37.5 \%$ \\
\hline & Cavo fuerte & $3.4 \%$ \\
\hline & Cavo extremo & $1.3 \%$ \\
\hline
\end{tabular}

\section{DISCUSIÓN}

Dentro del ámbito de la rehabilitación deportiva, la medicina del deporte y la biomecánica, cobra importancia el estudio de la huella plantar (podometria) y sus implicaciones dentro de la práctica deportiva. La fisioterapia desde su área de intervención aporta técnicas para la prevención, manejo y rehabilitación de las alteraciones del pie, que determinan su funcionalidad y la sintomatología.

El presente estudio permite poner en evidencia las relaciones entre la estructura corporal, la morfología del pie, sus alteraciones y su sintomatología asociada al dolor, que puede ser en reposo o durante el entrenamiento. Sin embargo, el contexto en el cual se desenvuelve la práctica deportiva en 
el departamento de Sucre, permite ampliamente ahondar en las limitaciones de los deportistas para realizar correctamente sus sesiones respetando los procesos de crecimiento que evolucionan naturalmente y que a la vez condicionan la capacidad osteomuscular de los atletas.

A esto se suma la poca utilización de parámetros confiables para el análisis de la información y la poca utilización de herramientas tecnológicas en la práctica deportiva o para la evaluación de los aspectos importantes que se deben tener en cuenta para iniciar los procesos deportivos en edades iniciales; es así que se considera pertinente, ahondar en estas líneas de investigación, dado la marcada prevalencia de escuelas de formación deportiva que inician intervenciones a tempranas edades bajo ningún parámetro de medición biomecánico y diagnostico que permita establecer la morfología osteomuscular de su sistema locomotor específicamente del pie y la sintomatología de los problemas más comunes asociadas a prácticas deportivas (futbol). Por esto, el trabajo que aquí se plantea revelar se convierte en una herramienta para la biomecánica deportiva, para el deporte, para rehabilitación y para la medicina deportiva.

Las comparaciones que hacen los autores en este estudio, se valoran de forma positiva al tratar de encontrar relaciones entre componentes morfológicos (IMC y \% de grasa) los cuales puede ser útiles a la hora de determinar las proporciones de las masas, las dominancias entre ellas y el índice musculo/ósea e índice adiposo/muscular para efectos de comprensión e interpretación de la escala de clasificación del índice de masa corporal. Sin embargo, para el caso que analizamos no es una medida totalmente exacta del tipo de estudio en el cual nos centramos y que vale la pena discutir para poder encontrar las convergencias respectivas entre variables morfológicas, de percepción y un macro índice corporal (IMC).

Es así que los resultados de este estudio en cuanto a la tipología del pie derecho se encontraron que el tipo predominante fue el pie cavo, seguido del pie normal y con menor prevalencia el pie plano normal. Por otra parte, la tipología de pie izquierdo predominante fue el pie cavo, seguido del pie normal y con menor prevalencia el pie cavo extremo y el plano. Estos datos coinciden con la investigación propuesta por Pérez, L, y Iglesias, M. Denominada prevalencia de alteraciones musculo esqueléticas en el pie infantil: Estudio preliminar en la cual se realizó una exploración a través de un podoscopio en bipedestación en carga relajada con las rodillas extendidas y ambos talones apoyados; además se valoró la posición del pie utilizando el Foot Posture Index, cuyo uso permite cuantificar el grado 
de pronación, supinación o neutralidad partir de la palpación de la cabeza del astrágalo, las curvas supra e infra maleolares laterales, la posición del calcáneo, la prominencia talo navicular, la congmencia del arco longitudinal interno, y la abducción o aducción del ante pie respecto al retropié.

Los resultados más llamativos fueron el 95\% tenía alterado el reparto de presiones de la huella, un $75 \%$ presentaba alguna alteración en la dinámica (la más frecuente excesiva pronación en la fase de propulsión) y el mismo porcentaje de niños llevaban el calzado de talla pequeña. Más de la mitad tenía una huella anormal y el pie excesivamente pronado. Un $40 \%$ presentaba dolor, el $35 \%$ tenía pie cavo y un $10 \%$ pie plano. El $32 \%$ tenía alguna patología de ante pie. El resto de alteraciones fueron menos frecuentes, estos datos coinciden con lo hallado en esta investigación tras los resultados arrojados a través de la evaluación con el método Herzco y el software kinovea.

Con relación al apartado anterior, los hallazgos significativos en cuanto al tipo de pie, muestran la prevalencia del pie cavo bilateral, situación que devela alteraciones en la morfología de esta estructura para la práctica deportiva. Estos resultados muestran la realidad de los deportistas que se someten a sesiones de entrenamiento desde tempranas edades dentro de procesos de formación deportiva no especializada, condiciones que no son tenidas en cuentas por los entrenadores para la administración de cargas y dosificación de ejercicios potencialmente lesivos que ponen en riesgo la aparición del dolor posterior a la práctica. En este sentido, el estudio que aquí se muestra tiene una aplicación práctica asertiva y de impacto, por medio del cual se podrán ajustar las condiciones iniciales de los niños que ingresan a las selecciones y escuelas de formación deportiva en la ciudad de Sincelejo, no solo en fútbol sino en otras modalidades. De esta forma, los resultados se convierten en una línea base y diagnostico que muestra la necesidad de aplicar la ciencia al deporte y de partir bajo condiciones de valoración y diagnóstico para prevenir lesiones y molestias en el sistema osteomuscular de los niños que entrenan y garantizar un entrenamiento basado en la evidencia con el mínimo riesgo de lesión posible y sin aparición de dolor luego de la práctica.

Otra de las aplicaciones prácticas del estudio, representa la oportunidad de insumo para los traumatólogos y ortopedas, al tratar de buscar y establecer métodos de diagnóstico en podología, a fin de poder recomendar y diseñar plantillas ajustadas a la morfología del pie del niño y corregir deficiencias o alteraciones encontradas en ella. Los datos mostrados ante- 
riormente muestran con un alto porcentaje de confiabilidad los hallazgos más significativos, sin embargo, es claro precisar a lo largo de la metodología y los métodos de recolección de información, así mismo de valoración y diagnóstico, procedimientos utilizando herramientas tecnológicas para mayor confiabilidad de los datos que pueden disminuir el margen de error en el trazado de ángulos de la localización del pie. Estos procesos pueden realizarse empleando métodos por Imagenología, los cuales ayudan a minimizar el grado de inconsistencias en la valoración y diagnóstico de los instrumentos utilizados. En futuros estudios que se puedan derivar de este, es preciso utilizar este tipo de herramientas para determinar exactamente otras medidas podo métricas basadas en métodos más objetivos, dado a la simplicidad de la técnica de medición y valoración del procedimiento que se utilizó, los datos pueden ser asumidos como confiables y representativos de la población objeto de estudio.

Aydog et al. (2005b) Obtuvieron resultados similares en futbolistas. Estos autores justificaron éste hecho explicando el uso de pie dominante en fútbol. El pie que más funciones realiza (el dominante) verá modificada su morfología por diferentes tensiones musculares y ligamentosas y acabará dando valores más altos en los diferentes métodos de medición. Así mismo, Aydog S, Tetik O, Demirel H y Doral M proponen que el hecho de ser deportista de alto nivel y estar sometido a un entrenamiento intenso, puede provocar también estos cambios estructurales en el sistema musculo esquelético y ligamentoso. Además, según Hamill et al. (1989), otra de las causas a las que se puede deber esta diferencia entre los pies, es a la actividad muscular del pie plano que se verá incrementada. Así, el pie que tiene más actividad o función, en este caso el pie derecho, mostraría una tendencia a aplanarse. Por lo tanto, además se podría considerar que el gesto técnico de pisar el balón y el de parar el balón con la zona del ante pie, gestos muy utilizados en fútbol sala, provocan un desplazamiento hacia arriba de la cabeza de los metatarsianos y consecuentemente un estiramiento de la fascia plantar, que puede dar una tendencia a pie plano. Esto difiere de la presente investigación debido a que el pie plano normal derecho obtuvo un $1.3 \%$ y en la tipología del pie izquierdo el plano normal obtuvo un $2.2 \%$ estando en menor porcentaje.

Otra investigación encontrada que difiere de los datos hallados en el presente estudio es la denominada tipificación de la huella plantar de escolares entre 6 y 8 años de edad de población urbana del municipio de pamplona, en la cual se aplicó el método Herzco y la toma de datos coincide 
con la metodología manejada en esta investigación, utilizando el papel fax, tablillas de podometría y alcohol. En el estudio Podométrico se evaluaron 72 niños, quienes representaron un total de 144 huellas plantares (dos por cada niño) que simbolizan el 100\% de la muestra; de los cuales 101 pies resultaron completamente planos, con un $70.1 \%$; 53 correspondieron al pie izquierdo plano con un $73 \%$ de los casos para este pie y 48 al pie derecho plano con el $66,7 \%$ de los casos para este pie. Resultados contrarios a los obtenidos en esta investigación, donde en mayor proporción se encontró un pie cavo bilateral y en menor prevalencia el pie plano.

Según estos autores, es de esperar que estas modificaciones agudas se hagan crónicas en el tiempo en una persona deportista que somete sus pies a una gran carga y esfuerzo, además se ha encontrado un aumento del porcentaje de la huella plantar mediante el método de Herzco. Estos datos sustentan los resultados hallados por los investigadores López J., Meana M., Vera F. y García, J (2006). donde encontraron el mismo tipo de pie en futbolistas de distintas categorías. Los resultados indicaban que la mayoría de los sujetos presentaban un pie normal y más valgo en el Angulo Tibio Calcáneo del pie derecho que del izquierdo; en cambio, al utilizar el método Herzco los resultados de morfotipo de pie se aproximan más hacia el cavo. Además, encontraron un pie cavo asociado a un valgo de calcáneo, de manera similar a lo que se ha encontrado en esta investigación, donde la tendencia habitual es hacia una dilatación de la huella en todas sus partes (antepié, mediopié y retropié), aunque según la disciplina y las actividades deportivas, se verán unas zonas más afectadas que otras.

Según Elvira J, Vera-García F, Meana M. y García J. en este estudio se empleó el índice de la huella descrito por Hernández (1990), los resultados comparativos de las huellas del pie derecho e izquierdo antes y después del ejercicio demuestran que las diferencias entre ambos pies son mínimas y en ningún caso estadísticamente significativas, además se logró observar las modificaciones en la huella plantar del pie dominante a consecuencia de las adaptaciones producidas por el entrenamiento de la marcha atlética. Estas modificaciones siguen la pauta encontrada en las respuestas del pie derecho, el pie predominantemente analizado en las adaptaciones puesto que todos menos uno eran diestros. Además de acuerdo a los resultados el $62,69 \%$, presentan un tipo de pie en la categoría de pie cavo. Sin embargo, a pesar de que parece demostrar una tendencia al pie cavo, no se puede concluir que sea debida exclusivamente a la práctica de la marcha atlética, puesto que se han encontrado valores entre el $47,73 \%$ y el $81,17 \%$ (entre 
pie normal y cavo fuerte). Este dato coincide con esta investigación pues se encontró en mayor prevalencia el pie cavo, así mismo, se halló asociación significativa entre tipología del pie derecho (Método Herzco) con la localización y el dolor podal.

En esta investigación se resuelve que en mayor proporción los niños tienen un pie con tendencia al cavo este resultado corrobora diversos estudios hechos en varios deportes, en los que se ha reportado la misma tendencia, la mayoría de autores sugiere que el cavo es consecuencia de la exigencia biomecánica, afirmación que sustenta lo encontrado en esta investigación Elvira, Riera, García y Roca.

Además, es importante recalcar que existe discrepancia en la literatura sobre la influencia del tipo de pie en el riesgo de lesión. Para algunos autores la estructura estática del pie no tiene necesariamente una relación con el comportamiento dinámico o una biomecánica anormal según los autores Nakhaee, Rahimi, Abaee, Rezasoltani y Kalantari; Razeghi y Batt , sin embargo otros estudios biomecánicos han demostrado que tanto la presencia de pie plano, como la de pie cavo, se relacionan con inadecuada absorción de los impactos Abian, Duran, Lara, Jimenez y Aguado,(2005) Abián Vicén, Alegre Durán, Lara Sánchez, Jiménez Linares y Aguado Jódar;(2005) que conlleva a una mayor presencia de lesiones como fracturas por estrés o tendinitis aquilianas. Por su parte Queen y cols, (2008) demostraron que el pie plano, presenta un incremento en al área de contacto medial, llevando consigo, un aumento de las fuerzas y presiones sobre el área, lo que hace sensiblemente mayor el riesgo de lesión en estos individuos que en los sujetos con pie normal. Simkin y cols,(1990) determinaron que los sujetos con un arco plantar alto, aumentaron el riesgo de fracturas tibiales y femorales por estrés, mientras que los individuos con pie plano, incrementaban el riesgo a fracturas metatarsianas.

El pie cavo que fue el de mayor prevalencia en este estudio, es un tipo de pie donde existe un aumento de la bóveda plantar o arco longitudinal, que puede estar asociado con una desviación en varo del calcáneo y retracción de los dedos (dedos en garra). Es una patología asintomática en la infancia y la edad juvenil, y la sintomatología se hace presente en la adolescencia y en el adulto, además, Revenga, sugiere que es importante apoyar este estudio con técnicas que evidencien la presencia del valgo o varo de calcáneo y sea veraz la información.

Este dato se sustenta desde la teoría que dice que la edad en la que se realiza el ejercicio es un factor significativo, pues el desarrollo de la masa 
ósea se produce durante el período de crecimiento óseo y no cuando éste se convierte en hueso maduro. Alcanzar un adecuado pico de masa ósea en la etapa de crecimiento puede ser un factor de prevención de futuras osteoporosis e incide significativamente en lo encontrado en esta investigación que aunque a pesar que los niños tienen alteraciones en los pies solo $15.9 \%$ de la población evaluado tuvo dolor en el último año y la causa más común fue traumática.

El pie cavo es muy frecuente en niños sanos y tiene carácter familiar. El dolor es menos habitual en el niño que en el adulto. Los motivos de consulta suelen ser por dificultad para calzarse, el rápido desgaste del borde externo de la suela y la deformación del zapato, la deformación del pie, el trastorno de la marcha (con los pies hacia dentro), o la notable frecuencia de las caídas y los esguinces de tobillo.

En este sentido, el bienestar físico del niño deportista se verá influenciado por diversos factores dentro y fuera de su práctica deportiva(futbol) y uno de ellos está relacionado con la morfología y tipología del pie, que aparte de proporcionarle funcionalidad y equilibrio natural al infante, le permitirá llevar a cabo tareas de locomoción más efectivas, y sin riesgo de alteraciones que le servirán para toda la vida y para y para su práctica deportiva:

Según Bermon, Z (2014) "El pie es el medio de transporte funcional del ser humano, por lo cual es conveniente analizar la dinámica y estática de esta estructura. El pie plano es una de las causas más frecuentes de consulta médica infantil."

\section{CONCLUSIONES Y RECOMENDACIONES}

La aplicación del modelo Herzco para definir la tipología del pie, resulta ser un método aceptado dentro de la comunidad científica, la Biomecánica y la rehabilitación deportiva, ya que permite de forma sencilla conocer el tipo de pie, su morfología y las alteraciones derivadas a la práctica deportiva del futbol. A partir de este estudio, se concluye que la mayoría de los niños presentaron un tipo de pie cavo bilateral con diferencias menores en ambos pies. Esto datos reflejan las posibles deficiencias morfológicas de los deportistas y las necesidades de intervención desde el ámbito biomecánico y de la rehabilitación para tratar de corregir los errores que puedan derivarse de malas prácticas, del mal uso del calzado y plantillas inadecuadas para el caso del futbol. Los resultados de este estudio ponen en evidencia la fiabilidad del método Herzco para detectar los tipos de pie y proyectar con base al diagnóstico a los deportistas dados a su fácil 
manejo y utilización para detectar anormalidades en la morfología del pie. Otros aspectos a destacar es el tipo de terreno en el cual se desenvuelven la mayoría de las prácticas deportivas, las canchas de arena tienen a ejercer mayor presión para el deportista obligando a la estructura podal a un movimiento de locomoción inadecuado que se traduce en un apoyo sobre el ante pie (punta de pie). Esta puede ser una de las posibles causas que ha modificado la tipología del pie en los niños, sumado al tipo de terreno, el tipo inadecuado de calzado y plantillas acordes a las necesidades podo métricas de los jugadores.

Líneas futuras de investigación en este campo, deben direccionarse a la selección de métodos más objetivos con tecnología de punta, que ayuden a la obtención de más información relacionada con la tipología del pie, los sistemas de apoyo y la incidencia de factores externos en la modificación de las estructuras podales. En este sentido, los medios tecnológicos y el uso de software libres no dejan de ser métodos fiables en la industria tecnológica biomecánica, sin embargo, los métodos manuales y científicamente validos (Herzco) representan alternativas sencillas de fácil manejo y con alto grado de confianza.

\section{REFERENCIAS BIBLIOGRÁFICAS}

Abian, J., Durán, L., Lara, A., Jiménez, L. y Aguado, X. (2000). (Producer). Fuerzas de reacción del suelo en pies planos y cavos. Podcast retrieved.; from www.uclm.edu/organos/Vic_Investigacion/gruposweb/BiomecanicaHumanaDeportiva/Curriculum.

Abián, J., Alegre, L., Lara, A., Jiménez, L. y Aguado, X. (2005). Fuerzas de reacción del suelo en pies cavos y planos. Archivos de medicina del deporte; 22(108), 285-292.

Aydog S, Ozcakar L, Tetik O, Demirel H, Hascelik Z, Doral M. (2005). Relation between foot arch index and ankle strength in elite gymnasts: a preliminary study. British Journal of Sports Medicine. 2005;39(3): e13

Aydog S, Tetik O, Demirel H. y Doral M. Differences in sole arch indices in various

sports. British Journal of Sport Medicine; 39(2)

Barajas-Ramón Y. Santana-Lobo FB. (2010). Características morfológicas de los deportistas con altos logros de las selecciones de levantamiento de pesas, voleibol y karate-do del departamento de Córdoba, Colombia. EFDeportes.com, Rev. Digital. Buenos Aires; 15(148).

Bermón Z. (2014) Tipificación de la huella plantar de escolares entre 6 y 8 años de edad de población urbana del municipio de pamplona. Rev. 
Movimiento Científico; 8(1): 44-52.

Cailliet, R. (2006). Anatomía funcional del pie y el tobillo. En: Cailliet, R. Anatomía Funcional Biomecánica. Edición 1. Editorial Marbán; 256-84.

Consejo de Organizaciones Internacionales de las Ciencias Médicas (CIOMS). (1993). Pautas Éticas Internacionales para la Investigación y Experimentación Biomédica en Seres Humanos, Ginebra: 53-56.

Elvira J, Vera-García F, Meana M. y García J. (2008). Análisis Biomecánico del apoyo plantar en la marcha atlética. Relación entre la huella plantar, ángulos de la articulación subastragalina y presiones plantares. European Journal of Human Movement; 20, 41-60.

Gómez, L. (2010). Características de la huella plantar en deportistas colombianos. Entramado ;12(6):158-67.

Hamill J, Bates B, Knutzen K, y Kirkpatrick G. Relationship between selected static an dynamic lower extremity measures. Clinical Biomechanics, 1989; 4(4): 217-225.

López J, Meana M, Vera F. y García, J. (2006). Respuestas, adaptaciones y simetría de la huella plantar producidas por la práctica de la marcha atlética. Rev de Ciencias de la Actividad Física y del Deporte España; 2(4): 21-26.

Mattus-Jimenez J, Pérez-Domínguez I. (1993). Adaptaciones en el apoyo del pie en niñas que practican danza. Rev. Mex Ortop. Traum. 1999; 13 (1): 77-81.

Nakhaee, Z., Rahimi, A., Abaee, M., Rezasoltani, A., y Kalantari, K. (2008). The relationship between the height of the medial longitudinal arch (MLA) and the ankle and knee injuries in professional runners. Rev. The Foot, 18 (2), 84-90.

Pérez, L, Iglesias, M. (2015). Prevalencia de alteraciones musculoesqueléticas en el pie infantil: Estudio preliminar/Prevalence of musculoskeletal disorders in children's foot: Preliminary study. Rev Intern De Ciencias Podológicas; 9(1): 1-16. Retrieved from http://search.proquest.com/docview/1645905435? accountid $=34487$

Queen, R., Mall, N., Nunley, J., y Chuckpaiwong, B. (2008). Difference in plantar loading between flat and normal feet during diferent athletic task. gaitpost2008;12(010).

República de Colombia. (1993). Ministerio de Salud. Decreto 11, Resolución No 008430. Santafé de Bogotá:

Simkin, A., Leichter, I., Giladi, M., Stein, M., y Milgrom, C. (1989). Combined effect of foot arch structure and an orthotic device on stress fractures. Rev. Foot \& Ankle International; 10(1), 25-29. 